monocytes with a panel of TLR ligands demonstrated strong TIMP-1 and IL-6 production following triggering with TLR8 agonists (ssRNA). TLR8-mediated TIMP-1 production was reduced in monocytes from a patient with a genetic TLR signalling defect or $\mathrm{HC}$ monocytes cultured with MyD88 inhibitory peptide. Furthermore, matrix assay of TLR8 stimulated monocytes also confirmed functional TIMP-1 secretion, as matrix metalloproteinase-1 activity was significantly inhibited.

Conclusions This study indicates a potential link between SSc serum factors and TLR signalling resulting in excessive TIMP-1 secretion by circulating monocytes from SSc patients.

\section{A2.21 TOLL-LIKE RECEPTOR DEPENDENT AUTOANTIGENS AND VESICLES FROM P. GINGIVALIS IN ANIMAL MODELS OF RA TO MODULATE COLLAGEN AND COLLAGEN ANTIBODY INDUCED ARTHRITIS}

doi:10.1136/annrheumdis-2013-203215.21

${ }^{2 B}$ Marklein, ${ }^{2} \mathrm{M}$ Sohn, ${ }^{1 Z}$ Konthur, ${ }^{2} \mathrm{~K}$ Grimm, ${ }^{3} \mathrm{G}$ Steiner, ${ }^{4} \mathrm{~F}$ Apparailly, ${ }^{2} \mathrm{G}-\mathrm{R}$ Burmester, ${ }^{1.2} \mathrm{~K}$ Skriner. 'Max-Planck-Institute for Molecular Genetics, Berlin, Germany; ${ }^{2}$ Charité University Medicine, Department of Rheumatology and Clinical Immunology, Humboldt University and Free University, Berlin, Germany; ${ }^{3}$ University Departments of the Vienna Biocenter. Department of Medical Biochemistry, Dr. Bohrgasse 9/4, A-1030 Vienna, Austria; " IINSERM, U844, Hôpital Saint Eloi, and Université Montpellier I, Montpellier, France

Background and Objectives A variety of animal models suggest that TLR signalling is important in the pathogenesis of RA and the generation of specific autoantibodies. This study was conducted with sera from patients with rheumatoid arthritis, as well as with arthritis animal models to identify identical autoantigens dependent on TLR7 and 9 in human and animal models for disease modifying use. Moreover TLR2 and TLR4 modulating bacterial vesicles from P. gingivalis containing PAD (Peptidyl-Arginine Deiminase) which is involved in citrullination was used to study the TLR2/4 in arthritis.

Materials and Methods Using protein philtre technology (28000 human protein philtre) the autoantigen profile of RA patients, mouse collagen and zymosan induced arthritis, as well as collagen and pristan induced arthritis in rats and TLR7, TLR9 deficient double-deficient and MyoD88 and Tir8 deficient mice of the MRLlpr/lpr background were obtained. Cationic liposomes transferring siRNAs, bacterial vesicles, lipomannan and LPS were used for the validation of their potential as therapeutic target in collagen or collagen antibody induced arthritis (CAIA).

Results We found 18 identical proteins targeted in human and animal situations of arthritis. These data identify mRNA binding hnRNPs proteins which are part of $\mathrm{P}$ bodies, stress granules and components of messenger RNA stability complex as well as CRP binding proteins as target molecules in mice, rats and humans with RA. Moreover, we found MyoD88 independent autoantigens which are not expressed in the thymus or proteins such as high mobility group box proteins 1 and 2 which are MyoD88 independent sensors of nucleic-acid-mediated innate immune responses. Systemic administration of siRNAs with cationic liposomes inhibiting expression of Toll dependent autoantigens overexpressed and targeted by autoantibodies in the human and mouse synovial tissue were used for the validation of their potential to inhibit collagen induced arthritis in $\mathrm{C} 57 \mathrm{BL} / 6 \mathrm{~J}$ mice. Moreover P. gingivalis vesicles containing the PAD induce a mild inflammatory response in the CAIA model of arthritis. $\mathrm{P}$. gingivalis LPS and lipomannan treated animals show a 80\% reduction of athrithis score compared to E coli LPS in a C57BL/6J CAIA model.

Conclusions Systemic blocking of common RNA or DNA binding proteins overexpressed in synovial target tissue appears to modify arthritis. $P$. gingivalis vesicles evolved the ability to intercept and undermine a subset of TLR2/4 signalling events for corrupting innate immunity and modulate RA.

\section{A2.22 TYROSINE PHOSPHORYLATION PATHWAYS IN MYELOID CELL-MEDIATED INFLAMMATORY DISEASES}

doi:10.1136/annrheumdis-2013-203215.22

M Kovács, T Németh, K Futosi, Z Jakus, C Sitaru A, Mócsai. Department of Physiology, Semmelweis University School of Medicine, Budapest, Hungary; Department of Dermatology, University of Freiburg, Germany

Background Tyrosine kinases are major therapeutic targets in cancer but their role in immune-mediated disease pathogenesis is less understood.

Methods Here we tested the role of tyrosine kinases and tyrosine kinase substrates in two autoantibody-mediated disease models, the $\mathrm{K} / \mathrm{BxN}$ serum-transfer arthritis and anti-collagen VII autoantibody-induced blistering skin diseases.

Results Mice genetically deficient of the Syk tyrosine kinase in the hematopoietic compartment were completely protected from autoantibody-induced arthritis and blistering skin disease. Mice lacking three myeloid-specific tyrosine kinases (Hck, Fgr and Lyn) or the tyrosine kinase substrate PLC $\gamma 2$ were also protected from autoantibody-induced diseases. In vitro, Src-family kinases were required for Syk activation by immune complexes and both were further required for activation of PLC $\gamma 2$. The Src-family-Syk-PLC $\gamma 2$ pathway mediated cytokine production by myeloid cells but not neutrophil or monocyte migration per se. Lineage-specific analyses revealed that during autoantibody-induced arhritis, this signalling pathway was required in myeloid cells (particular neutrophils) but not in mast cells or platelets. Finally, Src-family kinases were also required for activation of myeloid cells by monosodium urite crystals and their deficiency attenuated monosdium urate crystal-induced arthritis, indicating that the role of this signalling pathway is not restricted to autoantibody-mediated disease processes.

Conclusions Taken together, the Src-family-Syk-PLC $\gamma 2$ pathway is an important component of both autoantibody-mediated and autoantibody-independent inflammatory disease processes.

\section{A2.23 IMPAIRED NATURAL KILLER CELL FUNCTION IN DOCK8 DEFICIENCY}

doi:10.1136/annrheumdis-2013-203215.23

1.2MC Mizesko, 1.2PP Banerjee, ' ${ }^{2} \mathrm{~L}$ Monaco-Shawver, 1,2EM Mace, ${ }^{3} \mathrm{~W}$ Bernal, ${ }^{4} \mathrm{~J}$ Sawalle-Belohradsky, ${ }^{4 B}$ Belohradsky, ${ }^{4} \mathrm{~V}$ Heinz, ${ }^{5} \mathrm{AF}$ Freeman, ${ }^{3} \mathrm{KE}$ Sullivan, ${ }^{5} \mathrm{SM}$ Holland, ${ }^{6}$ TR Torgerson, ${ }^{7} \mathrm{~W}$ Al-Herz, ${ }^{8} \mathrm{~J}$ Chou, ${ }^{1,2} \mathrm{C} \mathrm{C}$ Hanson, ${ }^{4} \mathrm{MH}$ Albert, ${ }^{8} \mathrm{RS}$ Geha, ${ }^{4} \mathrm{ED}$ Renner, ${ }^{1,2} \mathrm{JS}$ Orange. 'Baylor College of Medicine; ${ }^{2} T e x a s$ Children's Hospital, Houston, TX: ${ }^{3}$ Children's Hospital of Philadelphia Research Institute, Philadelphia, PA, USA; 'University Children's Hospital, Ludwig Maximilian University, Munich, Germany; ${ }^{5}$ National Institute of Allergy and Infectious Diseases, National Institutes of Health Bethesda, MD, USA; 'University of Washington and Seattle Children's Hospital, Seattle, WA, USA; 'Kuwait University and Allergy and Clinical Immunology Unit, Kuwait City, Kuwait, ${ }^{8}$ Boston Children's Hospital, Boston, MA, USA

Introduction DOCK8 mutations are responsible for a rare autosomal recessive immunodeficiency syndrome associated with severe cutaneous viral infections, elevated IgE levels, environmental allergies, autoimmunity, and malignancy. DOCK8 activates CDC42, which is important for cell signalling and actin reorganisation. Natural killer cells play a vital role in tumour surveillance and defence against virally infected cells. NK cell function relies on the accumulation of actin at the NK cell immunologic synapse formed with target cells. Although abnormalities in $\mathrm{T}$ and $\mathrm{B}$ cell function have been described in DOCK8-deficient patients, the role of NK cells in this disease is poorly understood. 